\title{
Tecnologias Digitais Aplicadas ao Resgate de Patrimônio Cultural
}

\author{
Brenno Jansen Franco ${ }^{1}$, Lara Maria Almeida Ferreira ${ }^{1}$, Ivana Márcia Oliveira \\ Maia $^{1}$, Anselmo Cardoso de Paiva ${ }^{2}$ \\ ${ }^{1}$ Instituto Federal de Educação Ciência e Tecnologia do Maranhão - IFMA \\ Campus São Luís Monte Castelo, Coordenação Acadêmica de Design - CAD \\ São Luís MA - Brasil \\ ${ }^{2}$ Universidade Federal do Maranhão - UFMA \\ Núcleo de Computação Aplicada - NCA \\ São Luís MA - Brasil
}

brenno-b15@hotmail.com, laramarya@hotmail.com, ivana.maia@ifma.edu.br, anselmo.c.paiva@gmail.com

\begin{abstract}
The Scientific Initiation Program to high school students (PIBIC EM) of IFMA enables to students live experiences in the digital technology use applied to rescue culture. From this perspective, this article presents actions between the computer science and the study of traditional naval construction nontechnical to contributing to the preservation of this secular culture and the knowledge dissemination.
\end{abstract}

Keywords: computer graphics, culture, popular knowledge

Resumo. O programa de Iniciação Científica do Ensino Médio (PIBIC EM) do IFMA permite ao estudante vivenciar experiencias que investem no uso de tecnologia digital aplicada ao resgate da cultura. Nessa perspectiva, este artigo apresenta ações integradas entre a informática e o estudo das técnicas construtivas navais tradicionais populares com o objetivo de contribuir com a preservação dessa cultura secular e com a disseminação desse conhecimento.

Palavras chave: computação gráfica, cultura, conhecimento popular

\section{Introdução}

A oralidade, por muito tempo, constituiu-se como uma das principais ferramentas de propagação de ideias e ensinamentos transmitidos de geração em geração como uma forma de eternizar o conhecimento humano, tão importante quanto os registros gráficos, que desde a pré-história vêm sendo implementados, ilustrando a informação e contribuindo para a sua perpetuação. A partir do século XV, o interesse se volta também para a sua abrangência. Nesse período, Gutenberg contribuiu para a expansão da informação, que graças a imprensa, passou a atingir um número maior de pessoas. 
O crescente desenvolvimento da tecnologia proporciona um aumento substancial no acesso à informação e através das mídias digitais, que potencializam a comunicação e a participação social.

Este trabalho tem como foco o direcionamento de alunos do curso técnico de Comunicação Visual a nível médio em aplicar a tecnologia digital como recurso de resgate cultural das técnicas construtivas tradicionais populares das embarcações de madeira que ocorrem no litoral maranhense cujo processos construtivos e projetos, apesar de envolverem conhecimentos mais complexos, nunca eram registrados, nem sob forma de desenhos nem escritos. Eram passados de geração em geração ou para os aprendizes dos mestres apenas pela oralidade e pela observação direta.

Este tipo de transmissão de conhecimento sujeita a informação à inconsistência e ao desaparecimento. Dessa forma, neste trabalho o estudante atua como prosumidor, termo que segundo (Rapkewicz et al, 2014), descreve o fenômeno no qual se constata que o indivíduo não é apenas consumidor de conteúdos: Ele é, ao mesmo tempo, produtor de informação. $\mathrm{O}$ aluno torna-se um consumidor de conhecimentos que produz informações sobre o que consome e influencia outros consumidores. Nesse contexto, os envolvidos no trabalho adquirem o conhecimento e o aplicam no desenvolvimento do trabalho.

No ano de 1986 iniciou-se o projeto de pesquisa para o registro destas técnicas, que implicam em uma manifestação cultural tradicional e, ao mesmo tempo, meio de sobrevivência de grupos de profissionais litorâneos e ribeirinhos envolvidos com a pesca e transportes marítimos.

O Projeto de pesquisa “As Embarcações do Maranhão" (Andrès, 1998), único no gênero no Brasil, gerou o registro de dezessete modelos de embarcações, na forma de pranchas de desenho, registros dos métodos construtivos, cadastro de mestres construtores, cadernos de entrevistas, fotografias, dentre outras formas de registro.

Devido ao período da sua execução, o projeto acumulou suas informações em materiais perecíveis, como papeis vegetal, arroz, sulfite, tintas à base de água, ou seja, materiais muito vulneráveis ao tempo e às intempéries, de acesso restrito, o que compete com a importância das informações neles contidas.

No sentido de resgatar e propagar tais informações, este trabalho trata da aplicação de tecnologia digital na compilação dos desenhos técnicos e geração de imagens tridimensionais com animação gráfica. Esses artifícios, além de favorecerem a perpetuação dos conhecimentos, figuram como uma forma mais interessante e atual de apresentação, que chama e prende a atenção do observador, além de contribuir com resgate da cultura envolvida no processo.

\subsection{O Projeto "As Embarcações do Maranhão"}

O presente trabalho trata do resgate, preservação e divulgação da documentação técnica e científica produzida no período de 1986 a 1998, com a execução do projeto de pesquisa "Embarcações do Maranhão", de autoria do engenheiro Luiz Phelipe Andrès, com apoio da FINEP/Secretaria de Planejamento do Maranhão, com a finalidade de 
V Congresso Brasileiro de Informática na Educação (CBIE 2016)

Anais do XXII Workshop de Informática na Escola (WIE 2016)

resgatar as técnicas tradicionais populares de construção das embarcações típicas da navegação maranhense.

O projeto, que culminou com a publicação de livro homônimo, despertou o interesse para as embarcações maranhenses de madeira e suas peculiaridades. Foi o primeiro projeto de pesquisa nessa área no Maranhão.

$\mathrm{Na}$ pesquisa, foram identificados em todo o território maranhense dezessete modelos diferentes de embarcações típicas, atendendo às características da navegação em suas regiões, registrados conforme a Tabela 1 .

Tabela 1 - Documentação produzida no Projeto Embarcações do Maranhão.

Fonte: Maia, Projeto ADOC 2015

\begin{tabular}{|c|c|c|c|}
\hline Tipo & Quantidade & Material & $\begin{array}{c}\text { Situação quanto a } \\
\text { informatização }\end{array}$ \\
\hline Pranchas de desenho técnico & 67 & Papel vegetal - tinta nanquim & Não informatizado \\
\hline Pranchas de desenho artístico & 15 & $\begin{array}{c}\text { Papel Canson 180 g- tinta para } \\
\text { aerógrafo }\end{array}$ & Não informatizado \\
\hline $\begin{array}{c}\text { Volumes de relatórios da pesquisa } \\
\text { Fotografias preto/branco e } \\
\text { coloridas }\end{array}$ & 29 & $\begin{array}{c}\text { Papel sulfite 70g - encadernados } \\
- \text { capa dura }\end{array}$ & Não informatizado \\
\hline Negativos de Fotografias & 3.894 & Papel fotográfico & Não informatizado \\
\hline
\end{tabular}

Todo o material produzido no Projeto "Embarcações do Maranhão" é de propriedade do CVTEE (Centro Vocacional Tecnológico Estaleiro Escola do Maranhão) e seu conteúdo, até então, é para acesso restrito aos visitantes desta instituição, dada a natureza física do material original.

\subsection{A importância da cultura naval}

O Brasil tem extensa região costeira e desde antes da colonização faz uso do transporte marítimo e explora a pesca como como forte segmento econômico.

$\mathrm{Na}$ região nordeste, o estado do Maranhão apresenta o segundo maior litoral, o que define a forte aptidão para as atividades marinhas e ribeirinhas. Encontra-se entre os maiores pescadores nordestinos, embora as atividades pesqueiras no Maranhão ainda sejam praticadas de forma bastante primitiva com o uso de instrumentos artesanais. O litoral maranhense é bastante favorável à pesca devido fatores naturais como a extensão, a grande plataforma continental, estuários fluviais, marés e correntes marinhas. Todos os municípios costeiros praticam a pesca seja junto a costa com barcos geleiras e/ou barcos a remo.

A construção das embarcações de madeira, na sua grande maioria, há séculos atendem aos mesmos modelos e processos construtivos, vem apresentando considerável redução na sua produção. Segundo Andrès (1998) esse fenômeno se dá pela indiferença dos jovens em aprender e dar continuidade à construção artesanal, movidos pela falta de reconhecimento das várias profissões que envolvem o segmento, 
a dificuldade de adquirir matéria prima (madeira), baixos salários, condições insalubres e baixa procura, entre outros fatores.

O conhecimento que envolve a construção artesanal não era registrado e sempre repassado para os aprendizes por oralidade e observação direta, o que caracteriza fragilidade na recuperação e preservação desse conhecimento. Devido a ações nesse sentido, atualmente os processos construtivos dos dezessete modelos identificados como genuinamente maranhenses estão registrados, assim como os profissionais envolvidos e seus respectivos estaleiros.

\section{Métodos}

O trabalho, que está sendo desenvolvido no Campus São Luís Monte Castelo do Instituto Federal do Maranhão, conta com a participação de alunos do Curso de Comunicação Visual orientados por professora da instituição, presentes em todas as fases de sua execução. A primeira etapa do trabalho corresponde ao estudo básico sobre projeto de embarcações e sobre computação gráfica. $O$ treinamento faz parte da metodologia, de acordo com (Yuri et al, 2015) que afirma que não basta apenas entregar a ferramenta, pois os discentes precisam ter o entendimento de como realizar as ações determinadas.

Esta metodologia apresenta uma abordagem que escapa aos esquemas tradicionais e formais da metodologia científica. Afinal, o material que está sendo produzido é algo resgatado a partir das necessidades de preservação dos dados coletados em pesquisa ocorrida há aproximadamente trinta anos.

O trabalho foi planejado e está sendo executado a partir de dois enfoques que se complementam e que são absolutamente imprescindíveis para que os objetivos sejam concretizados: um enfoque na preservação da informação, para sistematizar o resgate dos documentos existentes a partir da aplicação de novas tecnologias digitais de registro e preservação arquivística e outro enfoque na qualidade da forma de apresentação da mensagem, que deve ser clara com recursos visuais atraentes e com objetivo de despertar o interesse pela construção naval tradicional.

Tendo em vista esta dupla abordagem, que deve ser compreendida enquanto uma totalidade, são utilizadas as seguintes técnicas: a) Técnicas usuais de levantamento e sistematização de documentos: localização e prospecção de fontes documentais (de cunho arquivístico e bibliográfico) no Estaleiro Escola do Maranhão, cuja biblioteca é detentora de todo o material reminiscente ao Projeto "Embarcações do Maranhão" e de autoria de Luiz Phelipe Andrès. b) Catalogação dos documentos, separados por modelos e forma de registro, para viabilizar à equipe o acesso e compreensão do processo construtivo e dos projetos de cada modelo de embarcação separadamente. c) Identificação do software compatível com a técnica de registro empregada. Para os desenhos técnicos, inclusive planos de linhas, elencou-se o AutoCAD (Autodesk, Inc.), com a preocupação de manter exatas as estruturas das pranchas de desenho originais. As pranchas de desenho foram escaneadas e as imagens importadas para o AutoCAD, onde sofreram interferências, no sentido de melhorar sua qualidade gráfica. Alguns desenhos foram vetorizados com auxílio do software CorelDRAW (Corel Corporation). Para os 
desenhos artísticos, a técnica utilizada foi o resgate da imagem original (produzido em papel de gramatura 180, com técnica de pintura por aerógrafo) utilizando o Adobe Photoshop (Adobe Systems), com tratamento da imagem para eliminar o desgaste e a ação do tempo, valorizando as formas e o colorido de origem.

Com base nos desenhos técnicos, fotografias e desenhos artísticos foi desenvolvida a modelagem tridimensional utilizando 3D Studio Max (Autodesk, Inc.) e a plataforma do Unity com câmera estática e efeitos de luz natural, com a possibilidade do observador percorrer todo o interior da embarcação e/ou visualizá-la em diferentes ângulos de observação. Esse recurso, acredita-se, é a forma de apresentação que pode atrair mais observadores e despertar interesse pelo tema. $\mathrm{O}$ material produzido também pode ser utilizado em jogos virtuais e material didático, para divulgação da cultura local e em atividades destinadas à discussão acerca do patrimônio histórico e cultural maranhense que poderá orientar uma política pública local com a finalidade de preservá-lo e conservá-lo.

Todo o material resultante deste trabalho será implementado em site de apresentação do Projeto "Embarcações do Maranhão" criado para o Centro Vocacional Tecnológico Estaleiro Escola do Maranhão e poderá ser utilizado na criação de jogos interativos explorando o tema e material didático para os cursos técnicos oferecidos na instituição.

\section{Resultados}

O trabalho, ainda em andamento, é desenvolvido no Programa de Iniciação Científica do Ensino Médio (PIBIC EM) do IFMA e envolve equipe formada por estudantes do curso médio técnico em Comunicação Visual.

Embora considerando a importância dos cadernos de relatórios do desenvolvimento da pesquisa, este material está sendo copiado por scanner e não requer atenção neste artigo.

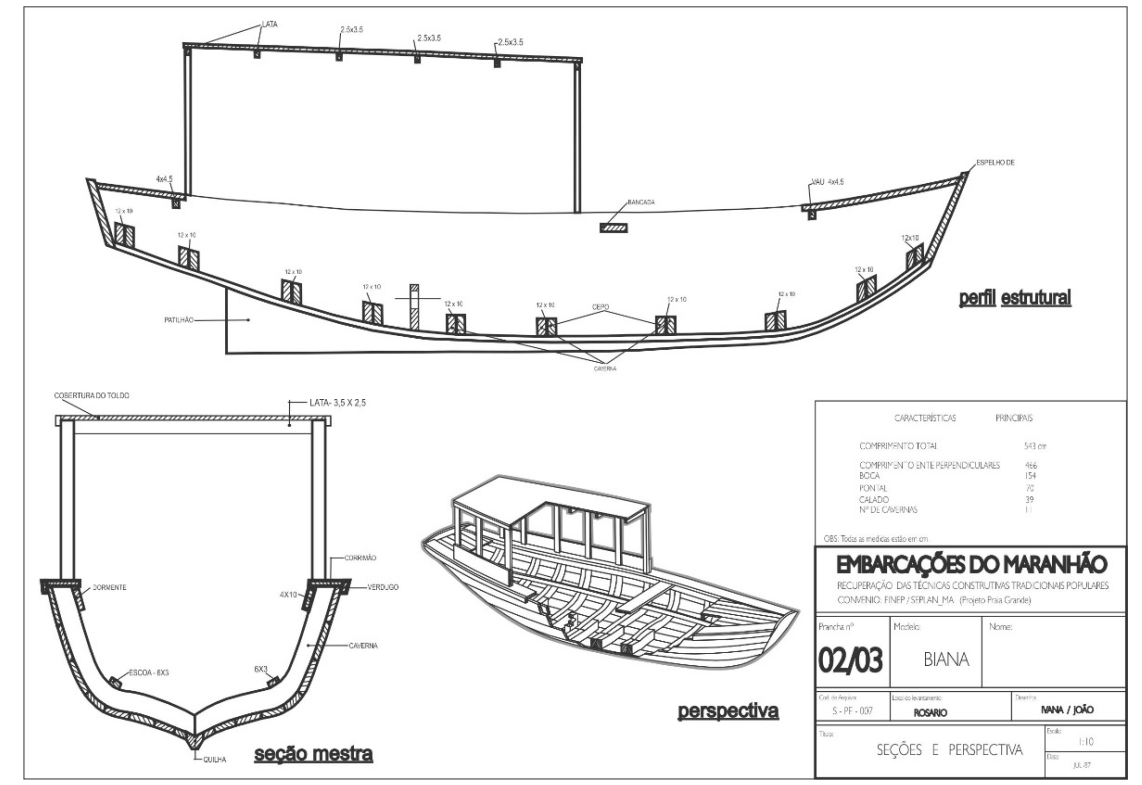

Figura 1: Prancha de projeto do modelo Biana 
O material que está sendo gerado é constantemente analisado sob a ótica do Design Gráfico, onde são observados aspectos referentes à Comunicação Visual no sentido de atenderem às demandas do objetivo do trabalho, mantendo-se fiel à documentação original. Com isso, associou-se recursos computacionais à cultura tradicional ao usar tecnologia digital para efetivar o resgate das pranchas de desenho técnico e artístico e gerar modelos tridimensionais (Fig. 2) que, além de ilustrarem com maior realismo as embarcações, permitem ao observador a visualização em ângulos de observação variados e a simulação de percorrer no interior das embarcações. Recursos que serão utilizados em variadas ferramentas de preservação da cultura naval e no desenvolvimento das atividades de pesca e transporte marítimo, como por exemplo site informativo, educacional, jogos interativos, material didático, entre outros.

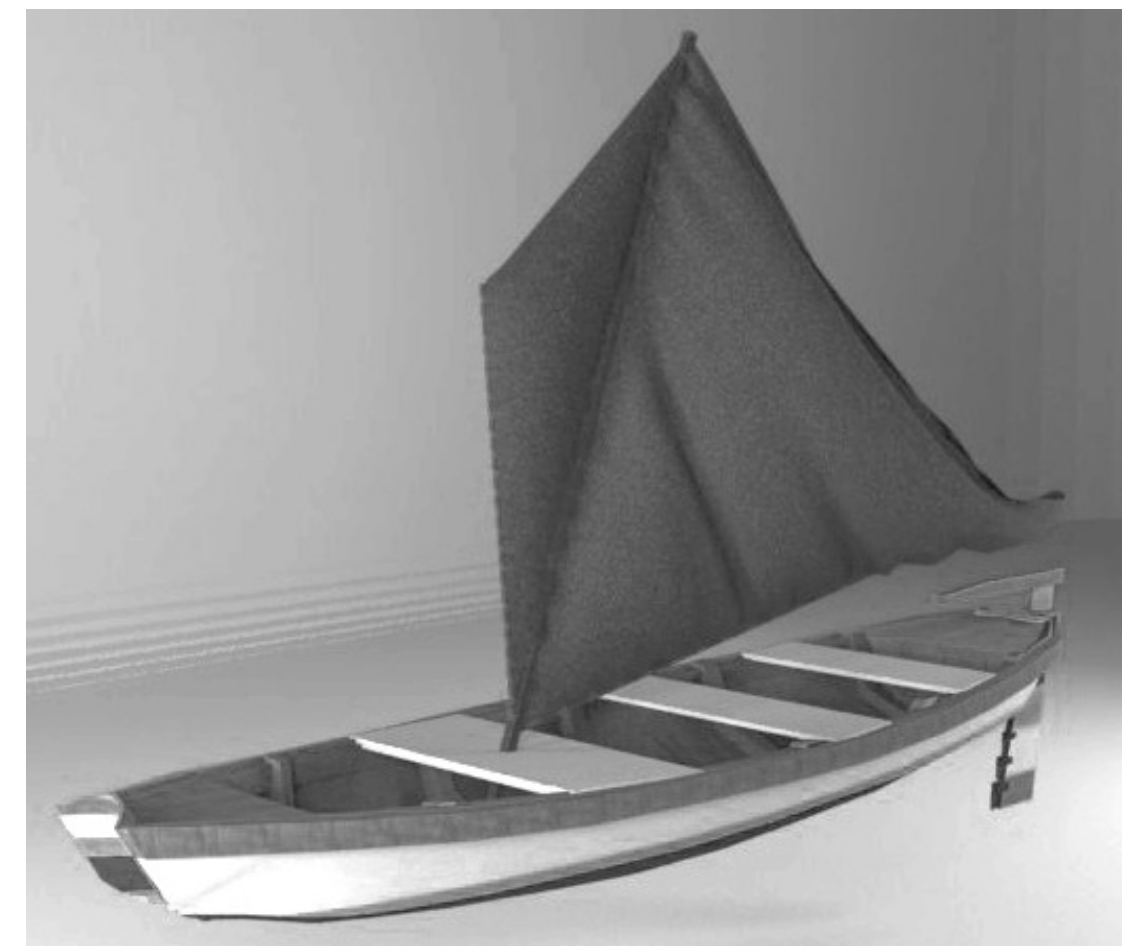

Figura 2: Modelagem tridimensional do modelo de embarcação denominado Biana

Dentre os modelos identificados, destaca-se a Biana. Trata-se de uma embarcação tradicional de pequeno a médio porte, de madeira, bastante utilizada no transporte e na pesca, sendo tanto apropriada para navegar em rios como no mar. É um dos modelos de maior incidência na ilha de São Luís. A preservação do modelo e processo construtivo da biana representa evitar o desaparecimento desse modelo, que por seu porte, compete com modernas embarcações de fibra de vidro e metal, porém com custo mais baixo de produção e facilidade de construção e manutenção em estaleiros sem muitos recursos. 


\section{Discussão}

A construção tradicional de embarcações, aos poucos, vem sendo substituída por processos construtivos e materiais desenvolvidos com recursos tecnológicos atuais, o que implica na contribuição da tecnologia para o desaparecimento das técnicas tradicionais populares, visto que os processos atuais envolvem materiais de mais fácil aquisição, menor tempo de construção e em algumas regiões, menores custos. A participação de estudantes da educação tecnológica em projetos de resgate cultural desperta para a importância da ação combinada entre as duas áreas - tecnologia e cultura- que não precisam se desenvolver separadas, nem são excludentes. A tecnologia da informação pode sim estar associada à preservação cultural e, como ferramenta desta, contribuir na formação de profissionais com consciência da importância da preservação da história e memória cultural. Estar atualizado tecnologicamente não significa segregar as antigas tecnologias regionais, mas associar o conhecimento empírico ao científico.

Espera-se que a ação combinada entre uma crescente compreensão dos dilemas relacionados à cultura e a aplicação dos recursos tecnológicos de computação gráfica no compartilhamento das informações, possam aproximar o público em geral ao amplo universo cultural.

\section{Conclusões}

A imersão dos estudantes de Comunicação Visual em processos de resgate cultural, como a projeção de um modelo de embarcação ou seu processo construtivo, permitiu que eles tivessem acesso e valorizassem as antigas manifestações culturais enquanto desenvolviam o projeto fazendo uso de tecnologia atual. Partindo do princípio que a experimentação de tecnologias digitais no âmbito da cultura proporcionou aproximação entre a tecnologia e a tradição, entende-se que este trabalho contribui com a universalização do conhecimento e com as modificações das relações entre as pessoas e suas memórias.

A velocidade e o raio de ação da tecnologia da informação potencializam a disseminação de conhecimento, ainda que este seja produzido em situações precárias e locais remotos. Segundo Ribeiro (2005), a informática não é apenas tecnologia, é uma linguagem. Fazer uso dessa linguagem despertou o interesse dos estudantes envolvidos no trabalho tanto no consumo do conhecimento como na produção de conteúdos que, explorados na esfera da computação gráfica, foram desenvolvidos no sentido de atrair e manter o interesse do observador no resgate e preservação das técnicas tradicionais populares de construção naval. Essa motivação deslocou os alunos de Comunicação Visual da sua prática comum e os lançou para um ambiente de descobertas em relação ao seu conhecimento e possibilidades de aplicações.

Dessa forma, conclui-se do trabalho, que a Tecnologia da Informação e a Comunicação Visual, quando aplicadas associadas aos projetos culturais e educacionais contribuem substancialmente à formação profissional e social do estudante e que a integração de saberes, melhora o entendimento acerca da área de TI e sua importância 
V Congresso Brasileiro de Informática na Educação (CBIE 2016)

Anais do XXII Workshop de Informática na Escola (WIE 2016)

na sociedade atual é pautada tanto no desenvolvimento da ciência, tecnologia e inovação como no resgate e preservação da cultura popular.

\section{Referência}

ANDRÈS, L. P. As Embarcações do Maranhão: Recuperação das Técnicas Construtivas Tradicionais Populares. São Paulo: Novo Horizonte, 1998.

MAGALHÃES, Y. L. S. F., SILVA, I. H. S., ALBUQUERQUE, C. H.; Capacitação para o aperfeiçoamento do conhecimento tecnológico aos alunos do Ensino Médio Anais do XXI Workshop de Informática na Escola (WIE 2015)

MAIA, I. M. O., Recuperação e Digitalização do Acervo do Projeto Embarcações do Maranhão. Projeto ADOC 03981/15, FAPEMA. Maranhão, 2015.

RAPKEWICZ, C. E., RODRIGUES, L., BAUERMANN, L.; Manifestações Expressivas na Inclusão Digital: Ações Integradas entre as Artes e a Informática na Educação de Jovens e Adultos

RAPKEWICZ , C. E., RODRIGUES L., BAUERMANN L. $3^{\circ}$ Congresso Brasileiro de Informática na Educação (CBIE 2014) 20a Workshop de Informática na Escola (WIE 2014). 
V Congresso Brasileiro de Informática na Educação (CBIE 2016)

Anais do XXII Workshop de Informática na Escola (WIE 2016) 